\title{
Improved Two Sample Revenue Guarantees via Mixed-Integer Linear Programming
}

\author{
Mete Şeref Ahunbay Adrian Vetta
}

March 2, 2021

\begin{abstract}
We study the performance of the Empirical Revenue Maximizing (ERM) mechanism in a single-item, single-seller, single-buyer setting. We assume the buyer's valuation is drawn from a regular distribution $F$ and that the seller has access to two independently drawn samples from $F$. By solving a family of mixed-integer linear programs (MILPs), the ERM mechanism is proven to guarantee at least .5914 times the optimal revenue in expectation. Using solutions to these MILPs, we also show that the worst-case efficiency of the ERM mechanism is at most .61035 times the optimal revenue. These guarantees improve upon the best known lower and upper bounds of .558 and .642, respectively, of Daskalakis and Zampetakis 4 .
\end{abstract}

\section{Introduction}

We study a primitive setting in revenue maximization: there is a single seller wishing to sell a single item to a single buyer, where the buyer's valuation for the item is drawn from a regular distribution $F$ on $[0, \infty)$. Further, we incorporate the now widespread supposition that the valuation distribution $F$ is unknown to the seller. Specifically, we present quantitative expected revenue guarantees when the seller is allowed access to two random, independently drawn sample valuations from $F$ before she selects a mechanism by which to sell the item.

When $F$ is known to the seller, Myerson [12] showed that the optimal mechanism the seller can implement is a posted price mechanism. In a posted price mechanism, the seller chooses a price $p$ and the buyer decides to either buy the item or not. Of course, under the implementation of such a mechanism, the buyer would purchase the item if and only if his valuation for the item is greater than $p$. Given this, the seller simply picks a price $p$ which maximizes her expected revenue. Formally, denoting the probability she sells the item for price $p^{\prime}$ as $1-F\left(p^{\prime}\right)$, the seller picks a price $p \in \max _{p^{\prime} \in \mathbb{R}_{+}} p^{\prime} \cdot\left(1-F\left(p^{\prime}\right)\right)$.

But what about the case when $F$ is unknown to the seller? When the seller has sample access to $F$, the natural approach is for the seller to assume the buyer's valuation distribution is given by the empirical distribution $\hat{F}$ induced by the set of samples; she may then simply implement the optimal mechanism of Myerson [12] using the empirical distribution. This method, called the Empirical Revenue Maximising (ERM) mechanism, provides surprisingly good performance guarantees even in the case of a single sample. Specifically, Dhangwatnotai et al. [6] showed that for the ERM mechanism just one sample suffices to give a $\frac{1}{2}$-approximation to the optimal revenue. Huang et al. [11] showed that this factor $\frac{1}{2}$ bound is tight for any deterministic mechanism. In 
contrast, Fu et al. [7] gave a probabilistic mechanism obtaining at least $\frac{1}{2}+5 \cdot 10^{-9}$ times the optimal revenue using a single sample.

On the other hand, another line of work studies the performance of the ERM mechanism with respect to sample complexity. This asks how many samples are necessary and/or sufficient to obtain a $(1-\epsilon)$-approximation of the optimal revenue, in expectation or with high probability. Dhangwatnotai et al. [6] noted that even in our simple setting, the ERM mechanism does not provide distribution independent polynomial sample complexity bounds; however, a guarded variant of the ERM mechanism which ignores an $\epsilon$ fraction of the largest samples does produce a $(1-\epsilon)$ approximate reserve price with probability $(1-\delta)$ given $\Omega\left(\epsilon^{-3} \cdot \ln \left(\frac{1}{\epsilon \delta}\right)\right)$ samples. Later, Huang et al. [11] showed that any pricing algorithm that obtains a $(1-\epsilon)$-approximation of the optimal revenue requires $\Omega\left(\epsilon^{-3}\right)$ samples, implying the factor $\epsilon^{-3}$ in the sample complexity result of [6] is tight. For more on the sample complexity of the ERM mechanism and its variants, see [1, 3, 5, 8, 9, 13, 10].

Motivated by the gap in our knowledge on sample complexity between the cases of a large number of samples and a single sample, Babaioff et al. 2] asked for revenue guarantees (in expectation) for a fixed number of samples $\geq 2$. Through a very rigorous case analysis they proved that, for two samples, the ERM mechanism breaks the factor $\frac{1}{2}$ barrier. Specifically, it guarantees at least .509 times the optimal revenue in expectation. Significant improvements in revenue guarantees were then provided by Daskalakis and Zampetakis [4]. They showed that with two samples a rounded version of the ERM mechanism obtains in expectation at least .558 times the optimal revenue. To achieve this they constructed a family of SDPs whose solutions provide lower bounds on the performance of the rounded ERM mechanism. Furthermore, through their primal solutions, they also showed that there exists a distribution of the buyer's valuation for which, with two samples, the ERM mechanism obtains in expectation at most .642 times the optimal revenue.

\subsection{Our Results}

In this paper, we study the ERM mechanism with two samples by building upon the optimization perspective of Daskalakis and Zampetakis [4]. However, rather than an SDP-based framework we use an MILP-based framework to inspect the performance of the ERM mechanism in the single seller, single buyer, single item setting. This approach may seem impractical at first glance, given the hardness of mixed-integer linear programming and the fact that, in general, there are no short certificates of optimality for solutions of MILPs. Our key technical contribution therefore is to present an MILP to bound the performance of the ERM mechanism and which, despite the presence of $\geq 1000$ binary variables, can be approximately solved in a reasonable amount of time with provably small error guarantees. This allows us to prove the ERM mechanism obtains at least .5914 times the optimal revenue. Furthermore, primal solutions to our MILPs show that there is a distribution $F$ for the buyer such that the ERM mechanism obtains at most .61035 times the optimal revenue. Consequently, we improve the lower bound on the revenue guarantee from .558 to .5914 and the upper bound from .642 to .61035. Note also that we specifically analyze the ERM mechanism rather than the rounded version of [4]. Thus, in particular, we improve the lower bound on guaranteed revenue under the ERM mechanism with two samples from the .509 of Babaioff et al. 2] to .5914. 


\subsection{Overview of Paper}

In Section 2 we present the problem of finding the worst case performance of the ERM mechanism with two samples. To motivate our MILP formulation, we prove the formulation of the problem in price space and quantile space are equivalent, and that for a given distribution $F$ of the buyer's valuation, the performance of the ERM mechanism can be calculated via an integral on $[0,1]^{2}$. In Section 3, we present a class of MILPs which approximate or underestimate this integral, allowing us to compute provable upper and lower bounds on the performance of the ERM mechanism in our setting. In Section 4, we show the resulting upper and lower bounds obtained by numerically solving these MILPs. Open problems and future directions are discussed in the conclusion.

\section{Preliminaries}

There are two agents: a seller and a buyer. The seller wishes to sell a single item to the buyer, whose valuation $v$ is drawn from a distribution $F$. To do so, the seller runs a posted price mechanism the seller commits to a price $p$, and the buyer can either take it or leave it. The buyer is utility maximizing, and his utility is quasilinear in payment. In particular, the buyer purchases the item if and only if $v \geq p$. Further, we make the standard assumption that the distribution of the buyer's valuation, $F$, is regular. A distribution $F$ on $\mathbb{R}_{+}$, given by its cumulative distribution function $F: \mathbb{R}_{+} \rightarrow[0,1]$, is called regular if its revenue curve $R(q)=(1-q) \cdot F^{-1}(q)$ is concave on $(0,1)$. The objective of the seller is to maximize her revenue, but the distribution $F$ is unknown to her. Instead she must select the posted price based upon (two) independently drawn samples from $F$.

\subsection{The Empirical Revenue Maximizing Mechanism}

Recall, by Myerson [12], when $F$ is regular the optimal revenue mechanism for the seller is a posted price mechanism with price $p \in \arg \max _{p^{\prime} \in \mathbb{R}_{+}} p^{\prime} \cdot\left(1-F\left(p^{\prime}\right)\right)$. However, to implement such a mechanism, the seller would need to know $F$. But, without knowledge of $F$, how could she implement such a mechanism given only two independent samples, say $s \geq t$, drawn from $F$ ? We assume the seller does the obvious and implements the Empirical Revenue Maximizing (ERM) mechanism. That is, she simply posts a price which maximizes her expected revenue with respect to the empirical distribution $\bar{F}$ she obtains via her two samples. Namely,

$$
\bar{F}(p)= \begin{cases}0 & p<t \\ 1 / 2 & t \leq p<s \\ 1 & s \leq p\end{cases}
$$

Thus the seller sets price $p=t$ if $s<2 t$, and sets price $p=s$ if $s>2 t$. If $s=2 t$, since we are interested in worst case revenue, we may assume that the seller picks $p \in\{s, t\}$ which minimizes $p \cdot(1-F(p))$. Denote the expected revenue from posting price $p$ by $r(p)=p \cdot(1-F(p))$. Next, let the bisample expected revenue $\psi_{F}(\cdot, \cdot)$ be defined as follows. When $s \geq t$, set

$$
\psi_{F}(s, t)=r(s) \cdot \mathbb{I}(s>2 t)+r(t) \cdot \mathbb{I}(s<2 t)+\min \{r(s), r(t)\} \cdot \mathbb{I}(s=2 t),
$$

and, when $s<t$, set $\psi_{F}(s, t)=\psi_{F}(t, s)$. Then the seller's revenue for implementing the ERM mechanism is exactly:

$$
\bar{r}_{F}=\int_{(s, t) \in \mathbb{R}_{+}^{2}} \psi_{F}(s, t) \cdot d F(s) \times d F(t)
$$


In turn, the optimal revenue for distribution $F$ is given by $r_{F}=\max _{p \in \mathbb{R}_{+}} p \cdot(1-F(p))$. In this paper, we are interested in providing lower and upper bounds for the relative performance of the ERM mechanism and the optimal mechanism, $\alpha=\inf _{F \mid F}$ is regular $\bar{r}_{F} / r_{F}$.

\subsection{Revenue Curves and the Quantile Space}

Following Daskalakis and Zampetakis [4], we will deriving our bounds on $\alpha$ via a reduction to a set of optimization programs for which we compute solutions. Importantly, however, we make a very different choice of variables. Specifically, we choose to work in the quantile space (i.e. with the revenue curve) rather than working in the price space (i.e. with the PDF/CDF of the distribution directly). Towards this end, note first that if $R$ is the revenue curve of the distribution $F$ then $R(q)=(1-q) \cdot F^{-1}(q)$ for any $q \in[0,1]$. Therefore, for each $q \in[0,1]$, the revenue curve provides the price inverse of $q$ :

$$
F^{-1}(q)= \begin{cases}R(q) /(1-q) & q \in[0,1) \\ \lim _{q^{\prime} \rightarrow 1^{-}} R\left(q^{\prime}\right) /\left(1-q^{\prime}\right) & q=1\end{cases}
$$

Via the price inverse, we may define the bisample revenue function $\phi_{R}(\cdot, \cdot)$ on $[0,1]^{2}$. To do this, if $(x, y) \in[0,1]^{2}$ and $x \geq y$, set

$$
\begin{gathered}
\phi_{R}(x, y)=R(x) \cdot \mathbb{I}\left[F^{-1}(x)>2 F^{-1}(y)\right]+R(y) \cdot \mathbb{I}\left[F^{-1}(x)<2 F^{-1}(y)\right] \\
+\min \{R(x), R(y)\} \cdot \mathbb{I}\left[F^{-1}(x)=2 F^{-1}(y)\right]
\end{gathered}
$$

If instead $x<y$, we symmetrically extend the function by setting $\phi_{R}(x, y)=\phi_{R}(y, x)$.

We want to write $\bar{r}_{F}$ as a double integral on $[0,1]^{2}$. By (2), this integral should have the form:

$$
\bar{r}_{R}=\int_{(x, y) \in[0,1]^{2}} \phi_{R}(x, y) \cdot d(x, y)
$$

We remark that there are several immediate advantages of such a choice of variables. First, in price space, the regularity constraints are highly non-linear; as shown by Daskalakis and Zampetakis [4], given a gridding of the real line, each regularity constraint is a degree three polynomial inequality. However, in quantile space, we can impose the regularity of the distribution with the following set of linear constraints:

$$
\forall x, y, \lambda \in[0,1], \quad R(\lambda x+(1-\lambda) y) \geq \lambda R(x)+(1-\lambda) R(y)
$$

Furthermore, working in quantile space, we only need to approximate an integral on $[0,1]^{2}$ with our optimization formulation, thereby avoiding the need to explicitly eliminate the tails of the distribution. Instead, the tiling of $[0,1]^{2}$ and normalisation of the optimal revenue naturally places an upper bound on $F^{-1}$ whenever $R(1)=0$.

Unfortunately, working in the quantile space also has disadvantages. While we can avoid nonlinearities in the regularity constraints, we will still need some quadratic constraints, and quadratic (or cubic) objective functions. In particular, both the feasible region and the objective function will be non-convex. Furthermore, we will work with an MILP formulation, using $\sim\left(\begin{array}{l}n \\ 2\end{array}\right)$ binary variables for a grid of $n$ intervals to handle degree 2 or degree 3 polynomial terms. Handling such a large set of binary variables for large $n$ will require care in how we set up our optimization programs. 


\subsection{The Validity of the Quantile Space Approach}

There is also a subtle technical complication we must address. Every regular distribution has a concave revenue curve. To get our approach of using revenue curves to work, we then want to write:

$$
\alpha=\inf _{F \mid F \text { is regular }} \bar{r}_{F} / r_{F}=\inf _{R \mid R:[0,1] \rightarrow \mathbb{R}_{+} \text {is concave }} \bar{r}_{R} / r_{R}
$$

where $r_{R}=\max _{q \in[0,1]} R(q)$. However, it is not the case that the integrals (2) and (44) are equal for each regular distribution, given our definition of regularity. For example:

Example 1. Consider the point mass at 1:

$$
F(x)= \begin{cases}0 & x<1 \\ 1 & x \geq 1\end{cases}
$$

Then $F^{-1}(q)=1$ for every $q \in(0,1]$, so the revenue curve is given $R(q)=1-q$. Here, the revenue curve is concave - in fact, affine. However, $\bar{r}_{F}=1$ while $\bar{r}_{R}=2 / 3$.

On the other hand, for any $\epsilon>0$, the uniform distribution on $[1,1+\epsilon]$ has revenue curve $R_{\epsilon}(q)=(1-q) \cdot(1+q \epsilon)$, a concave function. Furthermore, for any $\epsilon>0, \bar{r}_{F_{\epsilon}}=\bar{r}_{R_{\epsilon}}$, and in the limit $\epsilon \rightarrow 0, \bar{r}_{R_{\epsilon}} \downarrow 2 / 3$ as intended. This equivalence is caused by each distribution $F_{\epsilon}$ being continuous (i.e. having a probability density function). Consequently, to prove the equivalence of our formulation (5), we must prove a convergence result:

Theorem 2.1. Suppose that $R:[0,1] \rightarrow \mathbb{R}_{+}$is concave. Then there exists a sequence $\left(F_{n}\right)_{n \in \mathbb{N}}$ of continuous regular distributions such that the sequence of associated revenue curves $\left(R_{n}\right)_{n \in \mathbb{N}}$ converges uniformly to $R$ on $[0,1]$.

To prove Theorem 2.1, we need to prove several properties of concave, non-negative functions on $[0,1]$. The first result characterizes in a sense when $R$ fails to be the revenue curve of a continuous distribution:

Lemma 2.2. Suppose that $R:[0,1] \rightarrow \mathbb{R}_{+}$is concave (strictly concave), then $F^{-1}$ is non-decreasing (strictly increasing) on $[0,1)$. Moreover, $F^{-1}$ can only fail to strictly increase on a final segment: if for some $y<x$ in $[0,1)$ we have $F^{-1}(x)=F^{-1}(y)$, then for any $z \in[y, 1), F^{-1}(z)=F^{-1}(y)$.

Proof. Let $x, y \in[0,1)$ such that $y<x$. Then $x=\lambda \cdot y+(1-\lambda) \cdot 1$ for $\lambda=\frac{1-x}{1-y} \in(0,1)$. Now, as $R$ is concave, $R(x) \geq \lambda \cdot R(y)+(1-\lambda) \cdot R(1)$. However, $R$ is non-negative, so $R(1) \geq 0$. In particular, $R(x) \geq \lambda \cdot R(y)$. Because $\lambda=\frac{1-x}{1-y}$, we then have $\frac{R(x)}{1-x} \geq \frac{R(y)}{1-y}$.

To show the second statement, suppose that $F^{-1}(x)=F^{-1}(y)$. The case when $z \in(y, x)$ holds since $F^{-1}(\cdot)$ is non-decreasing. So suppose that $z \in(x, 1)$. Then $x=\lambda \cdot y+(1-\lambda) \cdot z$. If $F^{-1}(z)>F^{-1}(y)$ then $R(z)(1-y)>R(y)(1-z)$. However, by definition of $\lambda$, we have $(1-x)=\lambda(1-y)+(1-\lambda)(1-z)$. This implies that

$$
\begin{aligned}
R(y)(1-x) & =\lambda R(y)(1-y)+(1-\lambda)(1-z) R(y) \\
& <\lambda R(y)(1-y)+(1-\lambda)(1-y) R(z) \\
& =(1-y)(\lambda R(y)+(1-\lambda) R(z)) \\
& \leq(1-y) R(x) .
\end{aligned}
$$


Here the first inequality holds by the assumption $F^{-1}(z)>F^{-1}(y)$. The second inequality holds since $R$ is concave. This implies that $F^{-1}(x)>F^{-1}(y)$, a contradiction. Therefore, if $z \in(x, 1)$ then $F^{-1}(z)=F^{-1}(y)$.

This is sufficient to show that for any concave $R:[0,1] \rightarrow \mathbb{R}_{+}$, the set on which $\phi_{R}$ can be discontinuous has zero measure:

Corollary 2.3. Suppose that $R:[0,1] \rightarrow \mathbb{R}_{+}$is concave, and not identically zero. Then the set

$$
\hat{E}(R)=\left\{(x, y) \in[0,1]^{2} \mid R(x) \cdot(1-y)=2 R(y) \cdot(1-x)\right\}
$$

has Lebesgue measure zero.

Proof. We will show that $E(R)=\hat{E}(R) \cap[0,1)^{2}$ has Lebesgue measure zero, from which the result follows. Note that $E(R)$ is the set of $(x, y)$ such that $F^{-1}(x)=2 \cdot F^{-1}(y)$. We first show that $E(R)$ is a function mapping each $x$ to such $y 11$ Let $x, y, y^{\prime}$ be such that $y<y^{\prime}$, and $F^{-1}(x)=2 \cdot F^{-1}(y)=2 \cdot F^{-1}\left(y^{\prime}\right)$. Then $F^{-1}(y)=F^{-1}\left(y^{\prime}\right)$. Thus, by Lemma 2.2, $F^{-1}$ is constant on $[y, 1]$.

Now observe that, since $F^{-1}(x)=2 \cdot F^{-1}(y)$, we have $F^{-1}(x) \geq F^{-1}(y)$. If $x<y$ then $F^{-1}(x) \leq F^{-1}(y)$, by Lemma 2.2. Therefore, $F^{-1}(x)=F^{-1}(y)$. If instead $x \geq y$, then as $F^{-1}$ is constant on $[y, 1]$, we again have $F^{-1}(x)=F^{-1}(y)$. However, as $F^{-1}(x)=2 \cdot F^{-1}(y)$, this implies that $F^{-1}(y)=0$. Because $F^{-1}$ is constant on $[y, 1]$ we have $F^{-1}(1)=0$. So, as $F^{-1}$ is weakly increasing, $F^{-1}$ is identically zero on $[0,1]$. Therefore $R$ is identically zero on $[0,1]$, contradiction.

Consequently, for each $x \in[0,1]$, there exists at most one $y \in[0,1]$ such that $(x, y) \in E(R)$, that is, $E(R)$ is indeed a function. Let $d$ denote the domain of $E(R)$. By Lemma 2.2, $E(R)$ is increasing on its domain. Note that $R(x) /(1-x)$ is a continuous function, so $d$ is in fact an interval. Let $a=\inf d$ and $b=\sup d$. Let $y=\lim _{x \downarrow a} E(R)(x)$ and let $\bar{y}=\lim _{x \uparrow b} E(R)(x)$. For each $n$, divide $a=x_{0}$ and $x_{i}=a+i \cdot 2^{-n}(b-a)$, for $i \leq 2^{n}$. Also let $y_{0}=\underline{y}, y_{2^{n}}=\bar{y}$ and $y_{i}=E(R)\left(x_{i}\right)$, for $1 \leq i<2^{n}$. Then $E(R) \subseteq \cup_{i=1}^{2^{n}}\left[x_{i-1}, x_{i}\right] \times\left[y_{i-1}, y_{i}\right]=E_{n}(R)$ for each $n \in \mathbb{N}$. Furthermore, $E_{n}(R)$ has measure 0 in the limit $n \rightarrow \infty$, as $\mu\left(E_{n+1}(R)\right)=1 / 2 \cdot \mu\left(E_{n}(R)\right)$ for any $n \in \mathbb{N}$.

As an immediate result, we have the Riemann integrability of $\phi_{R}$.

Corollary 2.4. Suppose that $R:[0,1]^{2} \rightarrow \mathbb{R}_{+}$is concave. Then $\phi_{R}$ is Riemann integrable.

Proof. Since $R$ is continuous, the function is continuous on $[0,1]^{2} \backslash E(R)$. In particular, the function is discontinuous only on the subset of a set of Lebesgue measure zero. Therefore, by Lebesgue's criterion for Riemann integribility, the function is Riemann integrable.

So, the integral expression we have makes sense for any concave $R:[0,1]^{2} \rightarrow \mathbb{R}_{+}$. Next, we would like to obtain a sequence of probability density functions $f_{n}$, such that their associated revenue curves $R_{n}$ tend to $R$. We will do so by first defining the following:

Definition 1. Let $R:[0,1] \rightarrow \mathbb{R}_{+}$be a concave function. Then the $n$-interval piecewise approximation of $R$ is the function $R^{\mid n}:[0,1] \rightarrow \mathbb{R}_{+}$such that:

1. If $x=k / n$ for some $k \in \mathbb{N} \cup\{0\}$ and $k \leq n$, then $R^{\mid n}=R(x)$.

\footnotetext{
${ }^{1}$ Set-theoretically, a function is a set containing all input-output pairs.
} 
2. If $x=\lambda k / n+(1-\lambda)(k+1) / n$ for some $k \in \mathbb{N} \cup\{0\}$ and $k \leq n, \lambda \in(0,1)$, then

$$
R^{\mid n}(x)=\lambda R(k / n)+(1-\lambda) R((k+1) / n) .
$$

We are now ready to prove our convergence theorem:

Proof. (of Theorem 2.1) If $R$ is identically zero then the result immediately holds. So suppose not. Note that each $R^{\mid n}$ is again concave, and let $R_{n}(q)=R^{2^{n}}(q)+1 / n \cdot q(1-q)$. We first show that $R_{n}$ converges uniformly to $R$. Let $\epsilon>0$. If $x=k / 2^{m}$ for some $k, m$ then, for sufficiently large $n$, we have $R^{\mid 2^{n}}(x)=R(x)$. If not, as $R$ is concave on a compact set, $R$ is uniformly continuous. Hence $\exists \delta>0, \forall x, y,|x-y|<\delta \Rightarrow|R(x)-R(y)|<\epsilon / 2$. Then if $n>-\ln (\delta)$, there exists $k$ such that $k / 2^{n},(k+1) / 2^{n}$ are in the $\delta$-neighbourhood of $x$. Therefore, $\exists \lambda \in(0,1)$ such that $x=\lambda \cdot k / 2^{n}+(1-\lambda) \cdot(k+1) / 2^{n}$. So, by the definition of $R^{\mid 2^{n}}$,

$$
\begin{aligned}
\left|R^{\mid 2^{n}}(x)-R(x)\right| & =\left|\lambda R\left(2^{-n} k\right)+(1-\lambda) R\left(2^{-n}(k+1)\right)-R(x)\right| \\
& \leq \lambda \cdot\left|R\left(2^{-n} k\right)-R(x)\right|+(1-\lambda) \cdot\left|R\left(2^{-n}(k+1)\right)-R(x)\right| \\
& <\epsilon / 2 .
\end{aligned}
$$

Also for sufficiently large $n$, we have $2^{-n} \cdot x(1-x) \leq 2^{-n} \cdot \frac{1}{4} \leq \epsilon / 2$. Together this implies, for large $n$, that $\left|R_{n}(x)-R(x)\right|<\epsilon$. As $x$ was arbitrary, we indeed have $R_{n}$ converging uniformly $R$.

That $R_{n}$ corresponds to a distribution is immediate as $F_{n}^{-1}$ is differentiable everywhere on $(0,1)$ except a finite set. Furthermore, $F_{n}^{-1}$ is a bijection by Lemma 2.2. Thus, $F_{n}^{-1}$ has a differentiable inverse, $F_{n}$. In fact, for each $s$ on the image $F_{n}^{-1}((0,1))$, if $F(s)=q$ such that $F_{n}^{-1}(q)=s$, then $f(s)=\left(d F_{n}^{-1}(q) / d q\right)^{-1}$.

As a corollary, we conclude that the equivalence (5) does hold, showing that our decision to work in quantile space does not come at any cost regarding tightness of upper and lower bounds on $\alpha$ :

\section{Corollary 2.5.}

$$
\inf _{F \mid F \text { is regular }} \bar{r}_{F} / r_{F}=\inf _{R \mid R:[0,1] \rightarrow \mathbb{R}_{+} \text {is concave }} \bar{r}_{R} / r_{R}
$$

Proof. First let's show LHS $\leq$ RHS. Let $R$ be concave and non-negative on $[0,1]$ and let $R_{n}$ be as in the proof of Theorem 2.1. Since $R_{n}$ converges pointwise to $R, \phi_{R_{n}}$ also converges pointwise to $\phi_{R}$ almost everywhere. Also, both $\phi_{R_{n}}$ and $\phi_{R}$ are bounded above by the constant function $\sup _{q \in[0,1]} R(q)+1 / 2$. Therefore, by the Lebesgue dominated convergence theorem,

$$
\int_{(x, y) \in[0,1]^{2}} \phi_{R}(x, y)=\lim _{n \rightarrow \infty} \int_{(x, y) \in[0,1]^{2}} \phi_{R_{n}}(x, y)=\lim _{n \rightarrow \infty} \bar{r}_{R_{n}} .
$$

However, as $R_{n}$ is the revenue curve of a distribution which admits a probability density function, we have $\bar{r}_{R_{n}}=\bar{r}_{F_{n}}$. Further, by definition of $R_{n}$, for any $\epsilon$ there exists sufficiently large $n$ such that $r_{R}-\epsilon \leq r_{R_{n}} \leq r_{R}+\epsilon$. Together, this implies that for any concave and non-negative curve $R$ on $[0,1]$, there exists a sequence of distributions $F_{n}$ whose revenue efficiency converges to $\bar{r}_{R}$.

Next let's show LHS $\geq$ RHS. Take any regular distribution $F$. By Lemma $2.2, F$ can have a point mass only at the supremum of its support. Let $\rho$ be this supremum, and let $f(\rho)$ denote the 
probability the buyer's valuation is $\rho$. Note it is possible that $\rho=\infty$, but by regularity of $F$ that implies $f(\rho)=0$. In this case, note that the following change of variables still works:

$$
\int_{(s, t) \in[0, \rho)} \psi_{F}(s, t) \cdot d F(s) \times d F(t)=\int_{(x, y) \in[0,1-f(\rho)]^{2}} \phi_{R}(x, y) \cdot d(x, y) .
$$

If $f(\rho)=0$, then we are done. Else, we show that the remaining contributions of $\psi_{F}$ upper bound the contributions from $\phi_{R}$. If both samples equal $\rho$, then:

$$
\psi_{F}(\rho, \rho)=\phi_{R}(1-f(\rho), 1-f(\rho)) \geq \phi_{R}(x, y) \quad \forall x, y \in[1-f(\rho), 1]
$$

On the other hand, if one sample is $\rho$ and the other sample $t<\rho$, there are two possibilities. If $2 t>\rho$, then $\psi_{F}(\rho, t)=\phi(x, F(t))$ for any $x \in[1-f(\rho)]$. If instead $2 t<\rho$, then $\psi(\rho, t)=$ $R(1-f(\rho)) \geq \phi(x, F(t))$ for any $x \in[1-f(\rho)]$. We conclude that $\bar{r}_{F} \geq \bar{r}_{R}$.

\section{Approximation Programs}

The Riemann integrability of $\phi_{R}$ on $[0,1]^{2}$ suggests a possible optimization formulation for our problem. Given a gauge, we can try to find a concave and non-negative function $R$ on $[0,1]$, suitably constrained, such that an approximation of $\bar{r}_{R}$ is minimized. Here, we derive the forms of the optimization programs we evaluate, and prove their approximation properties. In Section 3.1 we present our main primal variables by formulating a class of quadratically-constrained programs whose solutions minimize approximations of (4) given some gauge on $[0,1]$. In Section 3.2 we provide a quadratic objective function for (4). Linearisation of the objective and the constraints then provides a family of MILPs suitable for searching for minimal distributions for the ERM mechanism with two samples, while proofs of well-behaviour of feasible solutions show that the value of these MILPs converge to $\alpha$ under gauge refinements. Motivated by this in Section 3.3 we formulate a cubic objective for (4) which allows us to obtain a family of MILPs whose values provide lower bounds on $\alpha$. In Section 3.4, we detail several considerations we employ to ensure that our MILPs are practically solvable and provide good bounds.

\subsection{Primal Variables}

To compute a Riemann sum of $\phi_{R}$ on $[0,1]^{2}$, we would first need to define a gauge on $[0,1]^{2}$. Here, we opt for the natural approach, defining a gauge on $[0,1]^{2}$ by considering product intervals arising from a gauge on $[0,1]$. In this line, suppose we divide the interval $[0,1]$ into subintervals of the form $I(i)=\left[q_{i}, q_{i+1}\right]$ for $1 \leq i \leq n$, where $q_{1}=0, q_{n+1}=1$, and $q_{i+1}>q_{i}$ for any $1 \leq i \leq n$. Also denote by $I(i, j)$ the product interval $\left[q_{i}, q_{i+1}\right] \times\left[q_{j}, q_{j+1}\right]$. Then we may rewrite integral (4) as:

$$
\begin{aligned}
\bar{r}_{R} & =\int_{(x, y) \in[0,1]^{2}} \phi_{R}(x, y) \cdot d(x, y) \\
& =\sum_{1 \leq i, j \leq n} \int_{(x, y) \in I(i, j)} \phi_{R}(x, y) \cdot d(x, y) \\
& =\sum_{1 \leq i \leq n} \int_{(x, y) \in I(i, i)} \phi_{R}(x, y) \cdot d(x, y)+2 \cdot \sum_{1 \leq j<i \leq n} \int_{(x, y) \in I(i, j)} \phi_{R}(x, y) \cdot d(x, y)
\end{aligned}
$$


We want primal solutions to our problems to describe approximately minimal value distributions for the buyer. One way to do so is to include variables that correspond to the values the revenue curve attains. Specifically, for $\left(q_{i}\right)_{1 \leq i \leq n+1}$, we will include variables $R\left(q_{i}\right)$. For notational convenience later on, let $\vec{R}$ denote the vector containing all $R\left(q_{i}\right)$.

Then each $R\left(q_{i}\right)$ corresponds to a value attained by a non-negative, concave function. This implies that the following constraints must hold:

$$
\begin{aligned}
R\left(q_{i}\right) \cdot\left(q_{i+1}-q_{i-1}\right) & \geq R\left(q_{i+1}\right) \cdot\left(q_{i}-q_{i-1}\right)+R\left(q_{i-1}\right) \cdot\left(q_{i+1}-q_{i}\right) & & \forall 1<i<n+1 \\
R\left(q_{i}\right) & \geq 0 & & \forall 1 \leq i \leq n+1
\end{aligned}
$$

Furthermore, we want $R$ to be normalized such that $\max _{q \in[0,1]} R(q)=1$. Unfortunately, this is non-trivial to implement linearly. So, instead, we constrain the set of revenue curves so that there exists $1 \leq O P T \leq n$ and $q^{*} \in\left[q_{O P T}, q_{O P T+1}\right]$ such that $R\left(q^{*}\right)=1$. By the concavity and non-negativity of $R$, this implies that:

$$
\begin{aligned}
R\left(q_{O P T}\right) & \geq q_{O P T} / q_{O P T+1} \\
R\left(q_{O P T+1}\right) & \geq\left(1-q_{O P T+1}\right) /\left(1-q_{O P T}\right)
\end{aligned}
$$

Furthermore, by concavity, $R(\cdot)$ should be weakly increasing before $q_{O P T}$ and weakly decreasing beyond $q_{O P T+1}$ :

$$
\begin{aligned}
R\left(q_{i+1}\right)-R\left(q_{i}\right) \leq 0 & & \forall 1 \leq i<q_{O P T} \\
-R\left(q_{i+1}\right)+R\left(q_{i}\right) \leq 0 & & \forall q_{O P T+1} \leq i<n
\end{aligned}
$$

We also model the indicator functions in (3) as binary variables:

Lemma 3.1. For any $(x, y) \in[0,1)$ such that $x>y$,

$$
\begin{aligned}
\phi_{R}(x, y)=\min _{w(x, y) \in\{0,1\}} & R(x) \cdot w(x, y)+R(y) \cdot(1-w(x, y)) \\
\text { subject to } & w(x, y) \cdot[R(x) \cdot(1-y)-2 R(y) \cdot(1-x)] \geq 0 \\
& (1-w(x, y)) \cdot[R(x) \cdot(1-y)-2 R(y) \cdot(1-x)] \leq 0
\end{aligned}
$$

Proof. If $R(x)(1-y)>2 R(y)(1-x)$ then the only feasible point is $w(x, y)=1$. This correctly sets $\phi_{R}(x, y)=R(x)$. Likewise, if $R(x)(1-y)<2 R(y)(1-x)$ then the only feasible point is $w(x, y)=0$, which correctly sets $\phi_{R}(x, y)=R(y)$. If $R(x)(1-y)=2 R(y)(1-x)$, then both $w(x, y)=1$ and $w(x, y)=0$ are feasible. This correctly sets $\phi_{R}(x, y)=\min \{R(x), R(y)\}$.

To compute a Riemann sum, we evaluate $w$ on a set $T$ of points in $[0,1]^{2}$ such that:

$$
\forall 1 \leq j<i \leq n, \quad \exists\left(\bar{q}_{i}, \bar{q}_{j}\right) \in T,\left(\bar{q}_{i}, \bar{q}_{j}\right) \in\left[q_{i}, q_{i+1}\right] \times\left[q_{j}, q_{j+1}\right] .
$$

This condition implies that each non-diagonal area element $I(i, j)$ for $1 \leq j<i \leq n$ contains a point where we evaluate $w$.

We include variables for the value $R$ attains on endpoints of intervals, but $w$ may be evaluated (in principle) anywhere on $I(i, j)$. Then for $(x, y) \in T$, to be able to impose constraints of the form (12) and (13) on $w(x, y)$, we find $R(x)$ and $R(y)$ by linear interpolation on $\vec{R}$. In particular, if $x \in\left[q_{i}, q_{i+1}\right]$, then:

$$
R(x) \cdot\left(q_{i+1}-q_{i}\right)=R\left(q_{i}\right) \cdot\left(q_{i+1}-x\right)+R\left(q_{i+1}\right) \cdot\left(x-q_{i}\right),
$$


and likewise for $R(y)$. So setting $\vec{w}$ to be the vector containing all $w\left(x_{\ell}, y_{\ell}\right)$, for each individual summand in (6) we may approximate

$$
\int_{(x, y) \in I(i, j)} \phi_{R}(x, y) \cdot d(x, y) \simeq A(i, j) \cdot f_{i j}(\vec{R}, \vec{w})
$$

where $A(i, j)=\left(q_{i+1}-q_{i}\right)\left(q_{j+1}-q_{j}\right)$ is the area of $I(i, j)$, for $1 \leq j \leq i \leq n$, and $f_{i j}$ is some function determined by our approximation scheme, homogeneous of degree one in $\vec{R}$.

This provides the form of our most general optimization formulation: we consider a set of gauges indexed by a set $J,\left(\vec{q}^{k}\right)_{k \in J}$, such that $\cup_{k \in J}\left[q_{O P T^{k}}^{k}, q_{O P T^{k}+1}^{k}\right]=[0,1]$, and find $R$ that minimizes our approximation of $\bar{r}_{R}$ by computing:

$$
\min _{k \in J} \min _{\vec{R}, \vec{w}} \sum_{1 \leq i \leq n} A_{k}(i, i) \cdot f_{i i}(\vec{R}, \vec{w})+2 \cdot \sum_{1 \leq j<i \leq n} A_{k}(i, j) \cdot f_{i j}(\vec{R}, \vec{w})
$$

subject to (17), (9), (10), (11), (12), (13)

$$
\begin{aligned}
& \vec{R} \in[0,1]^{n+1} \\
& \vec{w} \in\{0,1\}^{T}
\end{aligned}
$$

\subsection{Upper Bound: A Quadratic Formulation}

To derive an upper bound, we will need to find an approximately-minimal revenue curve. We consider a straightforward implementation of (14) to do this. For $n \in \mathbb{N}$, we take the uniform gauge given by $q_{i}=(i-1) / n$ for $1 \leq i \leq n+1$, and consider each case when the peak of the revenue curve is in $\left[q_{k}, q_{k+1}\right]$ for $1 \leq k \leq n$. To evaluate the Riemann sum, mark the midpoint of each interval:

$$
\bar{q}_{i}=\frac{q_{i}+q_{i+1}}{2}
$$

Then to approximate our Riemann integral, for each $I(i, j)$ we will evaluate the function $\phi_{R}$ at $\left(\bar{q}_{i}, \bar{q}_{j}\right)$. So we set:

$$
\begin{array}{ll}
f_{i i}=\frac{R\left(q_{i}\right)+R\left(q_{i+1}\right)}{2} & \forall 1 \leq i \leq n \\
f_{i j}=\frac{R\left(q_{i}\right)+R\left(q_{i+1}\right)}{2} \cdot w\left(\bar{q}_{i}, \bar{q}_{j}\right)+\frac{R\left(q_{j}\right)+R\left(q_{j+1}\right)}{2} \cdot\left(1-w\left(\bar{q}_{i}, \bar{q}_{j}\right)\right) & \forall 1 \leq j<i \leq n
\end{array}
$$

If we evaluate the resulting optimization problem, the constraints (9) and (10) tend to "chip off" the peak of the revenue curve in the primal solutions. This is unlikely to be a feature of an actual minimal revenue curve, so we will convert the constraints (9) and (10) into a single equality constraint, at the cost of increasing the size of the index set $J$ by one. First observe that

$$
\max \left\{R\left(q_{O P T}\right), R\left(q_{O P T+1}\right)\right\} \geq \max \left\{q_{O P T} / q_{O P T+1},\left(1-q_{O P T+1}\right) /\left(1-q_{O P T}\right)\right\}
$$

for any feasible solution $(\vec{R}, \vec{w})$. Let $\vec{R}^{*}=\max \left\{R\left(q_{O P T}\right), R\left(q_{O P T+1}\right)\right\}^{-1} \vec{R}$. Then for any $1 \leq j \leq$ $i \leq n$, by the homogeneity of $f_{i j}$ in $\vec{R}$ :

$$
\begin{aligned}
A(i, j) \cdot f_{i j}(\vec{R}, \vec{w}) & =\max \left\{R\left(q_{O P T}\right), R\left(q_{O P T+1}\right)\right\} \cdot A(i, j) \cdot f_{i j}\left(\vec{R}^{*}, \vec{w}\right) \\
& \geq \max \left\{\frac{q_{O P T}}{q_{O P T+1}}, \frac{1-q_{O P T+1}}{1-q_{O P T}}\right\} \cdot A(i, j) \cdot f_{i j}\left(\vec{R}^{*}, \vec{w}\right) \\
& \geq \frac{n-1}{n+1} \cdot A(i, j) \cdot f_{i j}\left(\vec{R}^{*}, \vec{w}\right)
\end{aligned}
$$


Now, $\vec{R}^{*}$ has either $R\left(q_{O P T}\right)=1$ or $R\left(q_{O P T+1}\right)=1$. So we consider imposing such an equality constraint in our optimization programs to normalise the maximum of the revenue curve, dropping the optimality constraints (9) and (10) from our optimization program. We are also able to drop the constraint (11), since it is implied by $R\left(q_{k}\right)=1, R \leq 1$, and the concavity constraints (7).

Finally, note that with the uniform gauge, $A(i, j)=1 / n^{2}$, for any $1 \leq j \leq i \leq n$. Thus, our Riemann sum minimization program is:

$$
\begin{aligned}
\hat{\alpha}(n)=\min _{1 \leq k \leq n+1} \min _{\vec{R}, \vec{w}} & \sum_{1 \leq i \leq n} \frac{1}{n^{2}} \cdot f_{i i}(\vec{R}, \vec{w})+2 \cdot \sum_{1 \leq j<i \leq n} \frac{1}{n^{2}} \cdot f_{i j}(\vec{R}, \vec{w}) \\
\text { subject to } & (7),(12),(13) \\
& R\left(q_{k}\right)=1 \\
& \vec{R} \in[0,1]^{n+1} \\
& \vec{w} \in\{0,1\}^{\left(\begin{array}{l}
n \\
2
\end{array}\right)}
\end{aligned}
$$

Intuitively, since the factor $(n-1) /(n+1)$ in (15) goes to 1 as $n$ grows large, this program should be able to approximate $\alpha$ :

Theorem 3.2. As $n \rightarrow \infty, \hat{\alpha}(n) \rightarrow \alpha$.

Before we prove this theorem, we emphasize an important monotonicity property of $w(x, y)$ : it is non-decreasing in the first argument and non-increasing in the second argument:

Lemma 3.3. Suppose that $R$ is concave and non-negative on $[0,1]$, and $w$ is determined as in Lemma 3.1. Then for any $x, y \in[0,1]^{2}$ such that $x>y$ :

(i) If $x^{\prime}>x$, then $w\left(x^{\prime}, y\right) \geq w(x, y)$.

(ii) If $x>y^{\prime}>y$, then $w\left(x, y^{\prime}\right) \leq w(x, y)$.

Proof. Now (i) can equivalently be stated $w(x, y)=1 \Rightarrow w\left(x^{\prime}, y\right)=1$, which we prove. Note that $R(x) /(1-x)$ is non-decreasing on $[0,1)$, which implies that $R(x) \cdot(1-y)-2 R(y) \cdot(1-x)$ is non-decreasing in $x$ on $[0,1]$. If it is the case that the constraint (12) does not bind or if $R(x) \cdot(1-y)-2 R(y) \cdot(1-x)$ strictly increases, then we are done. Else, it must be that $R\left(x^{\prime}\right)=$ $\left(1-x^{\prime}\right) R(x) /(1-x)<R(x)$ and so $\min \left\{R\left(x^{\prime}\right), R(y)\right\}=R\left(x^{\prime}\right)$. Therefore, $w\left(x^{\prime}, y\right)=1$. Likewise, (ii) can be equivalently stated as $w(x, y)=0 \Rightarrow w\left(x, y^{\prime}\right)=0$. Because $R(y) /(1-y)$ is increasing in $y$, we have $R(x) \cdot(1-y)-2 R(y) \cdot(1-x)$ is non-increasing in $y$ on $[0,1]$. From this, (ii) follows by an analogous argument.

These monotonicity properties of $w$ imply that only few $w\left(\bar{q}_{i}, \bar{q}_{j}\right)$ 's may be "misspecified". In particular, for some revenue curve $R$, the objective contributions $A(i, j) \cdot f_{i j}(\vec{R}, \vec{w})$ all underestimate their corresponding terms in 6 except for a vanishing fraction of product intervals $I(i, j)$ :

Lemma 3.4. Let $(\vec{R}, \vec{w})$ be a feasible solution of (16)), and let $R$ be a revenue curve agreeing with $\vec{R}$ on the gauge $\left(q_{i}\right)_{1 \leq i \leq n+1}$. Then for at least $\left(\begin{array}{l}n \\ 2\end{array}\right)-2 n+3$ many pairs $(i, j)$ such that $1 \leq j<i \leq n$, $w$ is a constant function on $I(i, j)$. In particular, for such pairs $(i, j)$ :

$\int_{(x, y) \in I(i, j)} \phi_{R}(x, y) \cdot d(x, y) \geq A(i, j) \cdot\left[\frac{R\left(q_{i}\right)+R\left(q_{i+1}\right)}{2} \cdot w\left(\bar{q}_{i}, \bar{q}_{j}\right)+\frac{R\left(q_{j}\right)+R\left(q_{j+1}\right)}{2} \cdot\left(1-w\left(\bar{q}_{i}, \bar{q}_{j}\right)\right)\right]$, with equality if $R$ is the linear interpolation of $\vec{R}$. 
Proof. We know that $w$ is determined as in Lemma 3.1 that $w$ and $\vec{w}$ may be taken to agree on $\left(\bar{q}_{i}, \bar{q}_{j}\right)_{1 \leq j<i \leq n}$, and that $w$ satisfies monotonicity by Lemma 3.3. Now, extend $w$ to points of the form $\left(q_{i}, q_{j}\right)_{1 \leq j \leq i \leq n+1}$ by setting $w\left(q_{i}, q_{i}\right)=0$ for any $1 \leq i \leq n$. We remark that this extension of $w$ will still satisfy monotonicity.

We now define a notion of constantness for $w$ on any $I(i, j)$ with $j<i$. We will say that the pair $(i, j)$ is 1 -definite if $w\left(q_{i+1}, q_{j}\right)=w\left(q_{i}, q_{j+1}\right)=1$, and 0 -definite if $w\left(q_{i+1}, q_{j}\right)=w\left(q_{i}, q_{j+1}\right)=1$. Else, by the monotonicity of $w$, it must be that $w\left(q_{i+1}, q_{j}\right)=1$ and $w\left(q_{i}, q_{j+1}\right)=0$; we call such a pair $(i, j)$ indefinite. Then, by the monotonicity of $w$, it holds that:

1. If $(i, j)$ is 1 -definite then $(i+1, j-1)$ is 1 -definite.

2. If $(i, j)$ is 0 -definite and $j+1<i-1$, then $(i-1, j+1)$ is 0 -definite.

3. If $(i, j)$ is indefinite then $(i+1, j-1)$ is 1 -definite, and if also $j+1<i-1$, then $(i-1, j+1)$ is 0-definite.

Therefore, for each $3 \leq k \leq 2 \cdot n-1$, the set of pairs $\{(i, j) \mid 1 \leq j<i \leq n$ and $i+j=k\}$ contains at most one indefinite pair. There are only $2 n-3$ such possible values of $k$. Now, if the pair $(i, j)$ is 1-definite, then

$$
\int_{(x, y) \in I(i, j)} \phi_{R}(x, y) \cdot d(x, y)=\int_{(x, y) \in I(i, j)} R(x) \cdot d(x, y) \geq A(i, j) \cdot \frac{R\left(q_{i}\right)+R\left(q_{i+1}\right)}{2},
$$

where the inequality holds due to concavity of $R$. If $R$ is an affine function on $I(i)$, then the inequality in fact holds with equality. A similar (in)equality holds if the pair $(i, j)$ is 0 -definite, which implies the result.

This result allows us to prove Theorem [3.2, and provide within the proof an explicit error estimate for $\hat{\alpha}(n)$ :

Proof. (of Theorem 3.2) For fixed $n \in \mathbb{N}$, let $R$ be the linear interpolation of a minimum primal solution of (16). Then,

$$
\int_{(x, y) \in I(i, j)} \phi_{R}(x, y) \cdot d(x, y)=\frac{1}{n^{2}} \cdot\left[\frac{R\left(q_{i}\right)+R\left(q_{i+1}\right)}{2} \cdot w\left(\bar{q}_{i}, \bar{q}_{j}\right)+\frac{R\left(q_{j}\right)+R\left(q_{j+1}\right)}{2} \cdot\left(1-w\left(\bar{q}_{i}, \bar{q}_{j}\right)\right)\right]
$$

for any $1 \leq j<i \leq n$, except at most $2 n-3$ many by Lemma 3.4. Also note that we may be misspecifying the contribution of areas of the form $(i, i)$. As $R \leq 1$, if we estimate $\phi_{R}(x, y)=$ $1+f_{i j}(\vec{R}, \vec{w})$ on such areas, we overestimate $\bar{r}_{R}$. Therefore, $\bar{r}_{R} \leq(5 n-6) / n^{2}+\hat{\alpha}(n)$. Since $\bar{r}_{R} \geq \alpha$, by the infimum property of $\alpha$, letting $n$ be sufficiently large such that $(5 n-6) / n^{2} \leq \epsilon$, we have $\hat{\alpha}(n) \geq \alpha-\epsilon$.

To show the upper bound on $\hat{\alpha}(n)$, let $\epsilon, \delta>0$. Let $R_{\delta}$ be a revenue curve such that $\bar{r}_{R_{\delta}} / r_{R_{\delta}} \leq$ $\alpha+\delta$, normalised such that $\max _{q \in[0,1]} R_{\delta}(q)=1$. Set $M(n)=\max _{q \in[0,1]} R_{\delta}^{\mid n}(q)$ for any $n$. Then $\lim _{n \rightarrow \infty} M(n)=1$. Also, since $R_{\delta}$ is regular, $M(n) \geq(n-1) /(n+1)$. Towards this end, we choose $n \in \mathbb{N}$ such that

$$
\frac{2}{n-1}+\frac{5 n-6}{n^{2}}<\epsilon
$$

Now, set $R_{\delta}^{n}=R^{\mid n} / M(n)$, the renormalized linear approximation to $R_{\delta}$. Then notice that an interval product $I(i, j)$ is definite for $R_{\delta}$ if and only if it is definite for $R_{\delta}^{n}$. Hence for any definite 
$I(i, j)$, the Riemann sum contribution of $\phi_{R_{\delta}} / M(n)$ is greater than that of $\phi_{R_{\delta}^{n}}$. If $I(i, j)$ is instead any interval, the Riemann sum contribution of $\phi_{R_{\delta}^{n}}$ at $I(i, j)$ is at most $1 / n^{2}$. Therefore,

$$
\begin{aligned}
\sum_{1 \leq i, j \leq n} & \frac{1}{n^{2}} \cdot \phi_{R_{\delta}^{n}}\left(\bar{q}_{i}, \bar{q}_{j}\right) \\
& =\frac{2}{n^{2}} \cdot\left[\sum_{\substack{1 \leq j<i \leq n \\
I(i, j) \text { definite }}} \phi_{R_{\delta}^{n}}\left(\bar{q}_{i}, \bar{q}_{j}\right)+\sum_{\substack{1 \leq j<i \leq n \\
I(i, j) \text { indefinite }}} \phi_{R_{\delta}^{n}}\left(\bar{q}_{i}, \bar{q}_{j}\right)\right]+\sum_{1 \leq i \leq n} \frac{1}{n^{2}} \cdot \phi_{R_{\delta}^{n}}\left(\bar{q}_{i}, \bar{q}_{i}\right) \\
& \leq \sum_{1 \leq i, j \leq n} \int_{\substack{(x, y) \in I(i, j) \\
R_{\delta} / M(n)}}\left(\bar{q}_{i}, \bar{q}_{j}\right) \cdot d(x, y)+\frac{4 n-6}{n^{2}}+\frac{n}{n^{2}} \\
& =\frac{n-1}{n+1} \cdot \sum_{1 \leq i, j \leq n} \int_{(x, y) \in I(i, j)} \phi_{R_{\delta}}\left(\bar{q}_{i}, \bar{q}_{j}\right) \cdot d(x, y)+\frac{5 n-6}{n^{2}} \\
& =\frac{n-1}{n+1} \cdot \bar{r}_{R_{\delta}}+\frac{5 n-6}{n^{2}} \leq \bar{r}_{R_{\delta}}+\frac{2}{n-1}+\frac{5 n-6}{n^{2}} \leq \alpha+\delta+\epsilon
\end{aligned}
$$

Here, the first inequality follows from Lemma 3.4 the second equality follows from homonegeity of $\phi$ in revenue curves, and the second inequality follows since $\bar{r}_{R_{\delta}} \leq 1$ by normalisation of $R_{\delta}$. However,

$$
\sum_{1 \leq i, j \leq n} \frac{1}{n^{2}} \cdot \phi_{R_{\delta}^{n}}\left(\bar{q}_{i}, \bar{q}_{j}\right) \geq \hat{\alpha}(n),
$$

as the sum equals the value of (16) when we plug in feasible solution $\vec{R}=\left(R_{\delta}^{n}\left(q_{i}\right)\right)_{1 \leq i \leq n+1}$. Combining these, we get the inequality

$$
\hat{\alpha}(n) \leq \sum_{1 \leq i, j \leq n} \frac{1}{n^{2}} \cdot \phi_{R_{\delta}^{n}}\left(\bar{q}_{i}, \bar{q}_{j}\right) \leq \alpha+\delta+\epsilon .
$$

Since our choice of $\delta$ is independent from our choice of $\epsilon$ and $n$, we may take $\delta \downarrow 0$, from which the theorem follows.

The convergence result suggests a natural optimization scheme to find an approximately minimal distribution - we linearize the terms of the form $R\left(q_{\ell}\right) \cdot w\left(\bar{q}_{i}, \bar{q}_{j}\right)$ in the objective and the constraints, adding in the constraints from the second-order Sherali-Adams lift of (16) that include such terms. In particular, we add in the constraints

$$
\begin{aligned}
R w(\ell, i, j) & \geq 0 \\
w\left(\bar{q}_{i}, \bar{q}_{j}\right)-R w(\ell, i, j) & \geq 0 \\
R\left(\bar{q}_{\ell}\right)-R w(\ell, i, j) & \geq 0 \\
-R\left(\bar{q}_{\ell}\right)-w\left(\bar{q}_{i}, \bar{q}_{j}\right)+R w(\ell, i, j) & \geq-1 \quad \forall 1 \leq j<i \leq n, \ell \in\{i, j\},
\end{aligned}
$$

where $R w(\ell, i, j)$ is a variable representing the product $R\left(\bar{q}_{\ell}\right) \cdot w\left(\bar{q}_{i}, \bar{q}_{j}\right)$. We then replace the product terms in constraints (12), (13) and in the objective with the corresponding linearized variable. Note that these constraints imply that $R w(\ell, i, j)=R\left(\bar{q}_{\ell}\right) \cdot w\left(\bar{q}_{i}, \bar{q}_{j}\right)$ whenever $w\left(\bar{q}_{i}, \bar{q}_{j}\right)$ is $\{0,1\}$-valued. Therefore, the mixed-integer LP formulation is exact. 
Finally, we impose the monotonicity constraints, implied by Lemma 3.3, even though these constraints are redundant for our formulation (cf. Section 3.4). This implies that our approximate MILP has the following form:

$$
\begin{aligned}
\min _{1 \leq k \leq n+1} \min _{\vec{R}, \vec{w}, R w} & \sum_{1 \leq i \leq n} \frac{1}{n^{2}} \cdot R\left(\bar{q}_{i}\right)+\sum_{1 \leq j<i \leq n} \frac{2}{n^{2}} \cdot\left(R w(i, i, j)+R\left(\bar{q}_{j}\right)-R w(j, i, j)\right) \\
\text { subject to } & (\underline{7}),(12),(13),[17 \\
& w\left(\bar{q}_{i}, \bar{q}_{j}\right) \leq w\left(\bar{q}_{i+1}, \bar{q}_{j}\right) \forall 1 \leq j<i<n \\
& w\left(\bar{q}_{i}, \bar{q}_{j}\right) \geq w\left(\bar{q}_{i}, \bar{q}_{j+1}\right) \forall 1 \leq j<i+1 \leq n \\
& R\left(q_{k}\right)=1 \\
& \vec{R} \in[0,1]^{n+1} \\
& \vec{w} \in\{0,1\}^{\left(\begin{array}{c}
n \\
2
\end{array}\right)} \\
& R w \in[0,1]^{2 \times\left(\begin{array}{l}
n \\
2
\end{array}\right)}
\end{aligned}
$$

\subsection{Lower Bound: A Cubic Formulation}

In this section, we construct an MILP with the explicit aim of obtaining lower bounds for $\alpha$. The MILP (18) does provide certifiable lower bounds for $\alpha$ by Theorem 3.2. However, the exponential nature of the problem kicks in before we can certify any significant improvement on the lower bound of $\simeq .558$ provided Daskalakis and Zampetakis [4].

We will work around this problem by considering a cubic program which, given a gauge, lower bounds the contribution of any area element. Handling the contribution of area elements on the diagonal will be straightforward, but the contributions from the off-diagonal area elements will require care. Towards this end, we will use Lemma 3.3, and lower bound this contribution conditional on the definiteness of the area element.

To construct such a lower bound program, we first need to fix our gauges: for the general formulation of the problem (14), we find a set of gauges $\left(\vec{q}^{k}\right)_{k \in I}$ with prescribed optimal intervals $\left[q_{O P T^{k}}^{k}, q_{O P T^{k}+1}^{k}\right]$ such that $\cup_{k \in I}\left[q_{O P T^{k}}^{k}, q_{O P T^{k}+1}^{k}\right]=[0,1]$. As evidenced by (15), we will want the freedom to pick $q_{O P T^{k}+1}^{k}-q_{O P T^{k}}^{k}$ small for each gauge $\vec{q}^{k}$ to minimize the loss from relaxing the optimality constraint to (9) and (10). To this end, for some $N \in \mathbb{N}$ "significantly larger" than $n$, we will set $J=\{1,2, \ldots, N\}$ and:

$$
\begin{aligned}
q_{O P T^{k}}^{k} & =\frac{k-1}{N} \\
q_{O P T^{k}+1}^{k} & =\frac{k}{N} .
\end{aligned}
$$

Then by (15), we expect degredations on the quality of the lower bound caused by the optimality constraints to be of order $\sim 1 / N$ as we impose larger $N$. Note that this only comes at a linear cost of having to compute $N$ MILPs.

Next, we need to decide on where to evaluate each $w\left(q_{i}, q_{j}\right)$. By Lemma 3.3, to decide on the definiteness of an area element $\left[q_{i}, q_{i+1}\right] \times\left[q_{j}, q_{j+1}\right]$ for $1 \leq j<i \leq n$, we need to check $w\left(q_{i+1}, q_{j}\right)$ and $w\left(q_{i}, q_{j+1}\right)$. Due to this constraint, we also need to assign a value to $w$ on $\left(q_{i}, q_{i}\right)_{1 \leq i \leq n+1}$. The defining constraints (12) and (13) become degenerate on such points. Instead we will opt to always 
fix $w\left(q_{i}, q_{i}\right)=0$, as such an assignment respects monotonicity and we wish to avoid adding even more binary variables.

We are now ready to derive lower bounds on the contribution of each area element. As promised, lower bounding the contribution of a diagonal area element is simple:

Lemma 3.5. Suppose $R:[0,1] \rightarrow \mathbb{R}_{+}$is concave and $I^{k}\left(O P T^{k}\right) \cap \arg \max _{q \in[0,1]} R(q)$ is non-empty. Then the following hold:

(i) If $i<O P T^{k}$, then $\int_{(x, y) \in I^{k}(i, i)} \phi_{R}(x, y) \cdot d(x, y) \geq A_{k}(i, i) \cdot\left(\frac{2 R\left(q_{i}^{k}\right)}{3}+\frac{R\left(q_{i+1}^{k}\right)}{3}\right)$.

(ii) If $i=O P T^{k}$, then $\int_{(x, y) \in I^{k}(i, i)} \phi_{R}(x, y) \cdot d(x, y) \geq 0$.

(iii) If $i>O P T^{k}$, then $\int_{(x, y) \in I^{k}(i, i)} \phi_{R}(x, y) \cdot d(x, y) \geq A_{k}(i, i) \cdot\left(\frac{R\left(q_{i}^{k}\right)}{3}+\frac{2 R\left(q_{i+1}^{k}\right)}{3}\right)$.

Proof. Observe that (ii) is trivial, so we show (i) and (iii). Let $R^{\prime}$ be the concave function on $[0,1]$, obtained by linear interpolation on $\left(R\left(q_{i}\right)\right)_{1 \leq i \leq n+1}$. Then $R \geq R^{\prime}$ on $[0,1]$, which implies that:

$$
\int_{(x, y) \in I^{k}(i, i)} \phi_{R}(x, y) \cdot d(x, y) \geq \int_{(x, y) \in I^{k}(i, i)} \min \left\{R^{\prime}(x), R^{\prime}(y)\right\} \cdot d(x, y) .
$$

$R^{\prime}$ is non-decreasing on $\left[0, q_{O P T^{k}}^{k}\right]$ and non-increasing on $\left[q_{O P T^{k}+1}^{k}, 1\right]$, and is affine on any $I^{k}(i)$. Thus for a lower bound for the revenue contribution from the product interval $I^{k}(i, i)$, we evaluate the minimum of two independent variables drawn from the uniform distribution on $R^{\prime}\left(I^{k}(i)\right)$, which implies that (i) and (iii) hold.

Next, the lower bounds on off-diagonal area elements, in turn, are dependent on whether the area element is definite:

Lemma 3.6. Suppose $R:[0,1] \rightarrow \mathbb{R}_{+}$is concave, $1 \leq j<i \leq n$, and $\left[q_{O P T^{k}}^{k}, q_{O P T^{k}+1}^{k}\right] \cap$ $\arg \max _{q \in[0,1]} R(q)$ is non-empty. Then the following hold:

(a) If the pair $(i, j)$ is 1-definite, then:

$$
\int_{(x, y) \in I(i, j)} \phi_{R}(x, y) \cdot d(x, y) \geq A_{k}(i, j) \cdot \frac{R\left(q_{i}^{k}\right)+R\left(q_{i+1}^{k}\right)}{2} .
$$

(b) If the pair $(i, j)$ is 0-definite, then:

$$
\int_{(x, y) \in I(i, j)} \phi_{R}(x, y) \cdot d(x, y) \geq A_{k}(i, j) \cdot \frac{R\left(q_{j}^{k}\right)+R\left(q_{j+1}^{k}\right)}{2} .
$$

(c) If the pair $(i, j)$ is indefinite and $i<O P T^{k}$, then:

$$
\int_{(x, y) \in I(i, j)} \phi_{R}(x, y) \cdot d(x, y) \geq A_{k}(i, j) \cdot \frac{R\left(q_{j}^{k}\right)+R\left(q_{j+1}^{k}\right)}{2} .
$$


(d) If the pair $(i, j)$ is indefinite and $j>O P T^{k}$, then:

$$
\int_{(x, y) \in I(i, j)} \phi_{R}(x, y) \cdot d(x, y) \geq A_{k}(i, j) \cdot \frac{R\left(q_{i}^{k}\right)+R\left(q_{i+1}^{k}\right)}{2} .
$$

(e) If the pair $(i, j)$ is indefinite, $i \geq O P T^{k}$ and $j \leq O P T^{k}$, then

$$
\int_{(x, y) \in I(i, j)} \phi_{R}(x, y) \cdot d(x, y) \geq A_{k}(i, j) \cdot \mathbb{E}[\min \{\underline{R}(x), \underline{R}(y)\} \mid(x, y) \in I(i, j)],
$$

where $\underline{R}$ is the minimum concave and non-negative function on $[0,1]$ satisfying (9) and (10).

Proof. If the pair $(i, j)$ is 1-definite, then

$$
\begin{aligned}
\int_{(x, y) \in I(i, j)} \phi_{R}(x, y) \cdot d(x, y) & =A_{k}(i, j) \cdot \mathbb{E}\left[R(x) \mid q_{i}^{k} \leq x \leq q_{i+1}^{k}\right] \\
& \geq A_{k}(i, j) \cdot \frac{R\left(q_{i}^{k}\right)+R\left(q_{i+1}^{k}\right)}{2},
\end{aligned}
$$

where the equality holds since $w(x, y)=1$ on $I(i, j)$ and the inequality holds by the concavity of $R$. This gives (a). The case (b) when $(i, j)$ is 0 -definite holds similarly.

Now suppose that the pair $(i, j)$ is indefinite. Then we use the inequality

$$
\int_{(x, y) \in I(i, j)} \phi_{R}(x, y) \cdot d(x, y) \geq A_{k}(i, j) \cdot \mathbb{E}[\min \{R(x), R(y)\} \mid(x, y) \in I(i, j)] .
$$

If $i<O P T_{k}$, then $R(y) \leq R(x)$ for $(x, y) \in I(i, j)$ as $R$ is concave and obtains its maximum at a point $q^{*} \geq q_{O P T^{k}}^{k} \geq x \geq y$. This implies (c) as:

$$
\begin{aligned}
A_{k}(i, j) \cdot \mathbb{E}[\min \{R(x), R(y)\} \mid(x, y) \in I(i, j)] & =A_{k}(i, j) \cdot \mathbb{E}\left[R(y) \mid q_{j}^{k} \leq y \leq q_{j+1}^{k}\right] \\
& \geq A_{k}(i, j) \cdot \frac{R\left(q_{j}^{k}\right)+R\left(q_{j+1}^{k}\right)}{2} .
\end{aligned}
$$

The case (d) where $j>O P T_{k}$ follows analogously. If neither case holds, then by the concave closure property we have $\underline{R} \leq R$. Therefore,

$$
A_{k}(i, j) \cdot \mathbb{E}[\min \{R(x), R(y)\} \mid(x, y) \in I(i, j)] \geq A_{k}(i, j) \cdot \mathbb{E}[\min \{\underline{R}(x), \underline{R}(y)\} \mid(x, y) \in I(i, j)] .
$$

Thus (e) holds.

This allows us to write a cubic expression which lower bounds the contribution from an offdiagonal area element to the revenue:

Corollary 3.7. Suppose $R:[0,1] \rightarrow \mathbb{R}_{+}$is concave, $1 \leq j<i \leq n$, and $\left[q_{O P T^{k}}^{k}, q_{O P T^{k}+1}^{k}\right] \cap$ $\arg \max _{q \in[0,1]} R(q)$ is non-empty. Let $f_{1 i j}(\vec{R}), f_{0 i j}(\vec{R}), f_{\text {cij }}(\vec{R})$ be respectively the lower bounds on the revenue contribution from the area element $I(i, j)$, conditional respectively on the pair $(i, j)$ being 1-definite, 0-definite or indefinite as in Lemma 3.6. Then:

$$
\begin{aligned}
\int_{(x, y) \in I(i, j)} \phi_{R}(x, y) \cdot d(x, y) \geq A_{k} & (i, j) f_{1 i j}(\vec{R}) w\left(q_{i+1}^{k}, q_{j}^{k}\right) w\left(q_{i}^{k}, q_{j+1}^{k}\right) \\
& +A_{k}(i, j) f_{0 i j}(\vec{R})\left(1-w\left(q_{i+1}^{k}, q_{j}^{k}\right)\right)\left(1-w\left(q_{i}^{k}, q_{j+1}^{k}\right)\right) \\
& \left.+A_{k}(i, j) f_{\iota i j}(\vec{R}) w\left(q_{i+1}^{k}, q_{j}^{k}\right)\right)\left(1-w\left(q_{i}^{k}, q_{j+1}^{k}\right)\right) \\
& +A_{k}(i, j) f_{\iota i j}(\vec{R})\left(1-w\left(q_{i+1}^{k}, q_{j}^{k}\right)\right) w\left(q_{i}^{k}, q_{j+1}^{k}\right)
\end{aligned}
$$


Proof. If the pair $(i, j)$ is 1-definite, then the RHS equals $A_{k}(i, j) f_{1 i j}(\vec{R})$, which by Lemma 3.6 is indeed a lower bound on the integral. The other cases follow similarly.

Note that the fourth term of the lower bound in Corollary 3.7 is redundant - it will equal zero for any integral solution for $\vec{w}$ by monotonicity. Still, the term allows us to gain some more strength in the LP relaxation of the program, so we retain it in our final formulation.

Given a gauge $\left(\vec{q}^{k}\right)$, a lower bound function $f_{i j}(\vec{R}, \vec{w})$ for each $1 \leq j \leq i \leq n$ is then provided by Lemma 3.5 and Corollary 3.7. To linearize the objective function, we again consider incorporating the relevant variables from the degree 3 Sherali-Adams lift of the problem, with their defining inequalities.

For the objective, we consider variables:

1. $w^{2}$ corresponding to terms of type $w\left(q_{i+1}, q_{j}\right) \cdot w\left(q_{i}, q_{j+1}\right)$,

2. $R w$ corresponding to terms of type $R\left(q_{\ell}\right) \cdot w\left(q_{i+1}, q_{j}\right)$ or $R\left(q_{\ell}\right) \cdot w\left(q_{i}, q_{j+1}\right)$, and

3. $R w^{2}$ corresponding to terms of type $R\left(q_{\ell}\right) \cdot w\left(q_{i+1}, q_{j}\right) \cdot w\left(q_{i}, q_{j+1}\right)$.

For $w^{2}$, the Sherali-Adams inequalities are then:

$$
\begin{array}{r}
\forall 1 \leq j<i \leq n, \\
-w\left(q_{i+1}, q_{j}\right)+w^{2}(i, j) \leq 0 \\
-w\left(q_{i}, q_{j+1}\right)+w^{2}(i, j) \leq 0 \\
w\left(q_{i+1}, q_{j}\right)+w\left(q_{i}, q_{j+1}\right)-w^{2}(i, j) \leq 1
\end{array}
$$

In turn, for $R w$, the Sherali-Adams inequalities are given:

$$
\begin{gathered}
\forall 1 \leq j<i \leq n, \forall \ell \in\{i, i+1, j, j+1\}, \forall(s, t) \in\{(i+1, j),(i, j+1)\}, \\
-R\left(q_{\ell}\right)+R w(\ell, s, t) \leq 0 \\
-w\left(q_{s}, q_{t}\right)+R w(\ell, s, t) \leq 0 \\
R\left(q_{\ell}\right)+w\left(q_{s}, q_{t}\right)-R w(\ell, s, t) \leq 1
\end{gathered}
$$

Finally, we have the Sherali-Adams inequalities for $R w^{2}$ :

$$
\begin{aligned}
& \forall 1 \leq j<i \leq n, \forall \ell \in\{i, i+1, j, j+1\},(21) \\
&-w^{2}(i, j)+R w^{2}(\ell, i, j) \leq 0 \\
&-R w(\ell, i+1, j)+R w^{2}(\ell, i, j) \leq 0 \\
&-R w(\ell, i, j+1)+R w^{2}(\ell, i, j) \leq 0 \\
&-w\left(q_{i+1}, q_{j}\right)+w^{2}(i, j)+R w(\ell, i+1, j)-R w^{2}(\ell, i, j) \leq 0 \\
&-w\left(q_{i}, q_{j+1}\right)+w^{2}(i, j)+R w(\ell, i, j+1)-R w^{2}(\ell, i, j) \leq 0 \\
&-R\left(q_{\ell}\right)+R w(\ell, i+1, j)+R w(\ell, i, j+1)-R w^{2}(\ell, i, j) \leq 0 \\
& R\left(q_{\ell}\right)+w\left(q_{i+1}, q_{j}\right)+w\left(q_{i}, q_{j+1}\right)-R w(\ell, i+1, j)-R w(\ell, i, j+1)-w^{2}(i, j)+R w^{2}(\ell, i, j) \leq 1
\end{aligned}
$$

For the defining constraints for $w$, (12) and (13), we linearize terms of the form $R\left(q_{\ell}\right) \cdot w\left(\bar{q}_{i}, \bar{q}_{j}\right)$ to $R w(\ell, i, j)$, coinciding with the previously defined $R w$ term whenever necessary. These terms 
have defining inequalities:

$$
\begin{aligned}
\forall 1 \leq j<i \leq n, \forall \ell \in\{i, j\} & \\
-R\left(q_{\ell}\right)+R w(\ell, i, j) & \leq 0 \\
-w\left(q_{i}, q_{j}\right)+R w(\ell, i, j) & \leq 0 \\
R\left(q_{\ell}\right)+w\left(q_{i}, q_{j}\right)-R w(\ell, i, j) & \leq 1
\end{aligned}
$$

Finally, we again impose the monotonicity constraints for $w$, despite their redundancy. This implies that our lower bounding MILP has the following form:

$$
\begin{aligned}
& \min _{k \in\{1,2, \ldots, N\}} \min _{\vec{R}, \vec{w}, w^{2}, R w, R w^{2}} \sum_{1 \leq i \leq n} A_{k}(i, i) \cdot f_{i i}(\vec{R}, \vec{w})+2 \cdot \sum_{1 \leq j<i \leq n} A_{k}(i, j) \cdot f_{i j}(\vec{R}, \vec{w}) \\
& \text { subject to (7), (9), (10), (11), (12), (13), (19), (20), (21), (22) } \\
& w\left(\bar{q}_{i}, \bar{q}_{j}\right) \leq w\left(\bar{q}_{i+1}, \bar{q}_{j}\right) \forall 1 \leq j<i<n \\
& w\left(\bar{q}_{i}, \bar{q}_{j}\right) \geq w\left(\bar{q}_{i}, \bar{q}_{j+1}\right) \forall 1 \leq j<i+1 \leq n \\
& w\left(q_{i}, q_{i}\right)=0 \forall 1 \leq i \leq n \\
& \vec{R} \in[0,1]^{n+1} \\
& \vec{w} \in\{0,1\}^{\left(\begin{array}{c}
n+1 \\
2
\end{array}\right)} \\
& w^{2}, R w, R w^{2} \geq 0
\end{aligned}
$$

\subsection{Technical Notes on Performance}

For computing an upper bound by searching approximate worst-case distributions and for computing a lower bound, we solve a number of mixed-integer linear programs. As we either approximate or lower bound a Riemann integral, more refined gauges provide better guarantees. On the other hand, mixed-integer linear programming is of course NP-hard in general, and for a gauge with approximately $\geq 45$ intervals, we have $\geq 1000$ binary variables. Therefore, we must select our gauge while considering whether the family of MILPs is practically solvable.

For both programs (18) and (23), we enforce inequalities corresponding to the monotonicity property of $w$ implied by Lemma 3.3. Even though such inequalities are redundant, we keep in mind that MILP solvers have to derive valid inequalities, and in general may not be able to deduce families of inequalities implied by the specific properties of the problem. Indeed, the inclusion of monotonicity inequalities for $w$ results in considerable speed-up for the programs (18) and (23).

Also, for a fixed number $n$ of intervals under consideration, (18) immediately provides a family of gauges. On the other hand, after selecting the number $N$ of optimal intervals, we still need to select gauges for (23) for each $k \in\{1,2, \ldots, N\}$. An immediate candidate is the "approximately uniform" gauge. For such a gauge, when $k=1$, we divide $[1 / N, 1]$ into $n-1$ equal size intervals. Likewise, when $k=N$, we divide $[0,1-1 / N]$ into $n-1$ equal size intervals. If instead $1<k<N$, we choose $m$ such that:

$$
m \in \arg \min _{1<\mu<n-1}\left|\frac{k-1}{N \cdot \mu}-\frac{N-k}{N \cdot(n-\mu-1)}\right|
$$

We then divide $[0,(k-1) / N]$ into $m$ equal size intervals, and $[k / N, 1]$ into $n-m-1$ equal size intervals. 


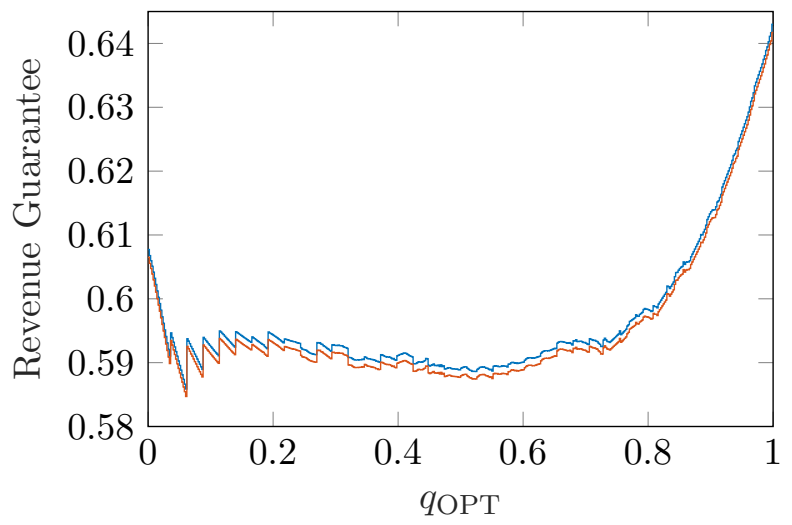

Figure 1: Computation of (23) with the approximately uniform gauge for $n=40$ and $N=500$. Values of solver output are shown in blue, while the lower bounds they imply after correcting for the tolerance of the solver are shown in orange. This computation implies a lower bound of .5847 via the lower bound for revenue when $q_{\mathrm{OPT}} \in[.06, .062]$.

While straightforward, this choice of gauge is problematic. To illustrate the issue, a computation of (23) with the approximately uniform gauge for $n=40$ and $N=500$ at $99.8 \%$ relative tolerance is shown in Figure 1. As seen in the figure, the approximately uniform gauge results in "jagged" behaviour for derived lower bounds when the revenue curve attains its maximum on (approximately) $[0, .2]$. The upwards kinks occur roughly when $k \rightarrow k+1$ causes $m \rightarrow m+1$. This implies that, for some initial segment of $[0,1]$, the quality of our lower bounds improve when we add more intervals in the segment $[0,(k-1) / N]$. As evidenced by Figure 1, somehow smoothing the jagged behaviour of the lower bound curve would allow us to improve our lower bound on the revenue of the ERM mechanism.

So we consider a modification of the approximately uniform gauge, square weighing the gauge on $[0,1 / 2]$. In particular, for $k<N / 2$, we instead choose $m$ such that

$$
m \in \arg \min _{1<\mu<n-1}\left|\frac{k-1}{N \cdot \mu^{2}}-\frac{N-k}{N \cdot(n-\mu-1)^{2}}\right| .
$$

Unfortunately, using this square-weighted gauge results in considerable slowdown of computations, when $k \lesssim N / 10$. For this reason, we lower the relative efficiency guarantees of our solver when $k \leq N / 10$. This results in a jump "discontinuity" in our computed revenue guarantees, but this effect is not strong enough to overpower improvements on our final lower bound due to choice of gauge.

For a comparison of the quality of lower bounds provided by the approximately uniform gauge and the (partially) square-weighted gauge, we show in Figure 2 a computation of (23) with the square-weighted gauge for $n=40$ and $N=500$, running our solver at $99.8 \%$ relative tolerance for $k>50$ and at $99 \%$ relative tolerance for $k \leq 50$. As seen, the derived lower bounds are smoothed on the initial segment of $[0,1]$ by the weighing, and despite the jump in the lower bounds due to change in tolerance at $k=50$, the quality of the lower bounds we obtain increase. The reason for why such a weighing works is unknown to us; indeed, we found the square-weighing rule by trial-and-error. 


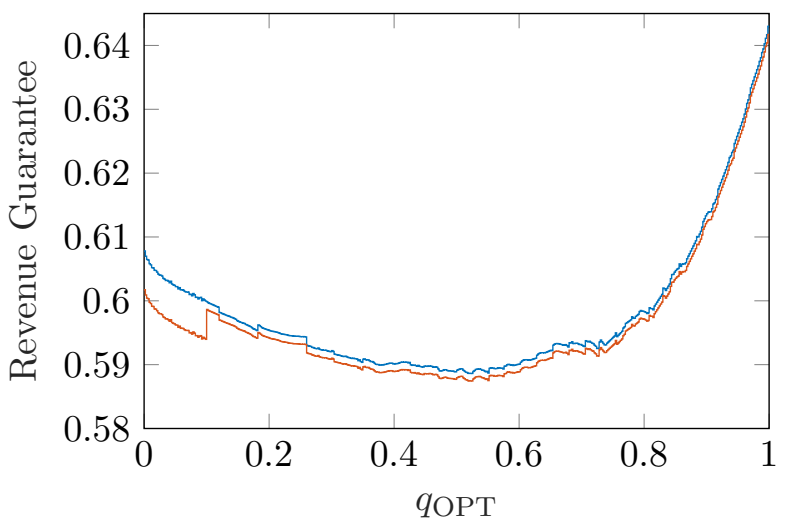

Figure 2: Computation of (23) with the square weighted gauge for $n=40$ and $N=500$, presented as in Figure 1. The square weighted gauge smoothes our lower bound estimates on $[0,1 / 2]$, providing a greater lower bound of .5874 .

\section{Results: Lower and Upper Bounds}

We are now ready to present lower and upper bounds on the performance of the ERM mechanism with two samples. We compute (18) and (23) using MATLAB + CPLEX as our MILP solver of choice 2 . We compute (18) for $n=80$, obtaining an approximate conditiona 3 minimum expected revenue curve. Each computation for $k \in\{1, \ldots, 81\}$ also provides us with an approximately minimal distribution; given primal solution $(R, w, R w)$ to (18) for $k$, we consider the minimum concave function $R_{k}$ such that $R_{k}(j / n)=R(j / n)$ for any $j=\{0,1, \ldots, 80\}$. By numerically evaluating the integral (44) in Mathematica for each such $R_{k}$, we obtain upper bounds on the performance of the ERM mechanism.

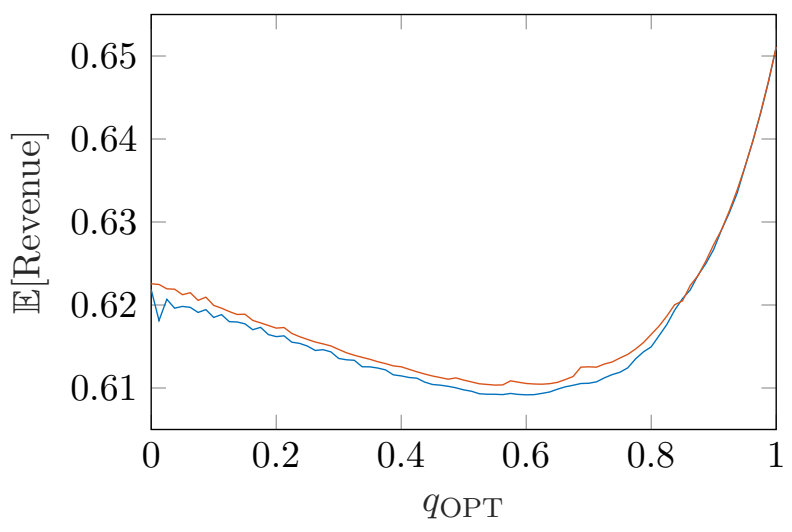

Figure 3: Results of computation of (18) for $n=80$. The values of (18) conditional on $q_{O P T}$ is shown in blue, while upper bounds on the performance of the ERM mechanism obtained from primal solutions are shown in orange.

The results of this computation is shown in Figure 3. Numerically computing the integral

\footnotetext{
${ }^{2}$ Our code is available at https://meteahunbay.com/files/code-twoSampleMILP.zip

${ }^{3}$ On $\arg \max _{q \in[0,1]} R(q)$.
} 
4 for each primal solution we obtain, the internal error estimates provided by Mathematica are $\leq 10^{-6}$ for each integral approximation. Via these computations, we find that our primal solution for $n=80$ and $q_{\mathrm{OPT}}=44 / 80$ provides a regular revenue curve for which the ERM mechanism obtains $\leq .61035$ times the optimal revenue. Furthermore, we are able to inspect the form of minimal distributions themselves. Figure 4 shows the form of such distributions - it appears they are closely approximated by piecewise linear functions on at most three intervals (3-piecewise linear functions).
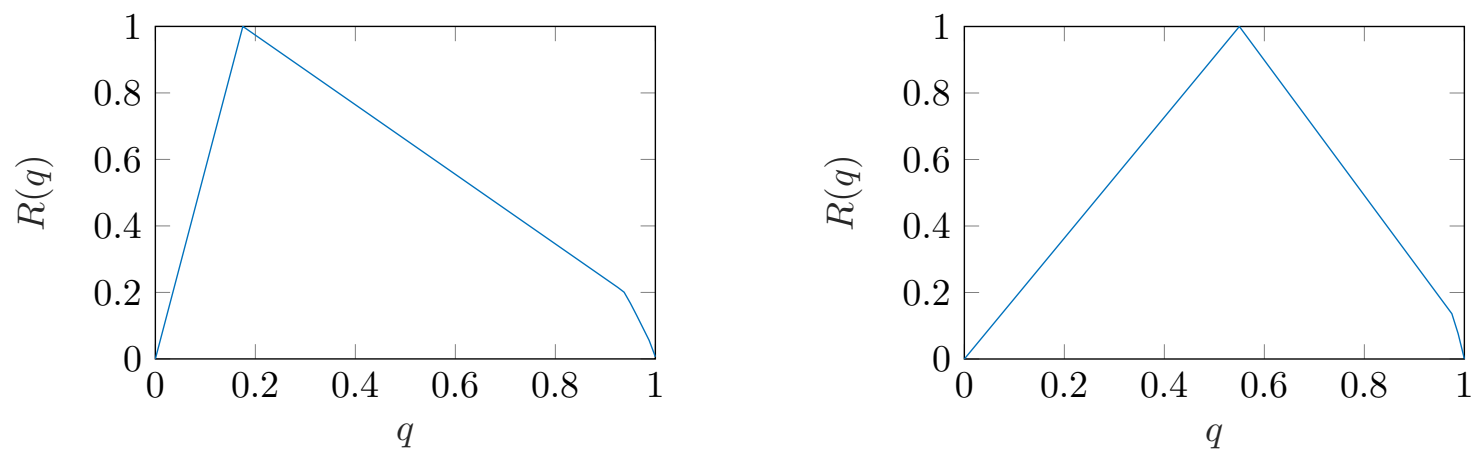

Figure 4: Revenue curves of approximately minimal distributions (conditional on $q_{O P T}$ ) obtained from (18) for $n=80$ and $k=15,45$.

This motivates us to inspect the efficiency of the ERM mechanism for piecewise linear functions on $\leq$ three intervals. Note that any such function $R$, normalised such that $\max _{q \in[0,1]} R(q)=1$, is the minimum concave function that contains $(0,0),\left(q_{O P T}, 1\right),\left(q_{2}, r_{2}\right),\left(1, r_{3}\right)$ in its hypograph for some $q_{O P T}, q_{2}, r_{2}, r_{3} \in[0,1]$. Given such $q_{O P T}, q_{2}, r_{2}, r_{3}$, we denote by $R\left(\cdot \mid q_{O P T}, q_{2}, r_{2}, r_{3}\right)$ the minimum concave function whose hypograph contains $(0,0),\left(q_{O P T}, 1\right),\left(q_{2}, r_{2}\right),\left(1, r_{3}\right)$.

Given $q_{O P T}$, the minimum performance of the ERM mechanism for functions of the form $R\left(\cdot \mid q_{O P T}, q_{2}, r_{2}, r_{3}\right)$ is given by:

$$
\eta\left(q_{O P T}\right)=\min _{q_{2}, r_{2}, r_{3}} \int_{(x, y) \in[0,1]^{2}} \phi_{R\left(\cdot \mid q_{O P T}, q_{2}, r_{2}, r_{3}\right)}(x, y) \cdot d(x, y)
$$

For $q_{O P T} \in\{0,1 / 80,2 / 80, \ldots, 1\}$ we attempt to approximate $\eta\left(q_{O P T}\right)$ by grid search, evaluating the minimum in (24) for

$$
\begin{array}{ll}
q_{2}=k / 80 & 71 \leq k \leq 80 \\
r_{2}=k / 1000 & 0 \leq k \leq 300 \\
r_{3}=k / 1000 & 0 \leq k \leq 18, k \in \mathbb{Z} .
\end{array}
$$

Note that we do not grid the entire cube $[0,1]^{3}$. We instead choose to restrict the bound of our grid search by the form of our primal solutions for (18). This is because we have found that the optimization problem for $\eta\left(q_{O P T}\right)$ is very poorly behaved. In particular, solutions obtained by searching over a coarse grid and then improving via gradient descent have provided greater (by $\gtrsim .01)$ upper bounds than simply searching on a fine grid. In addition, to keep computation costs low we have opted to restrict our search space. 


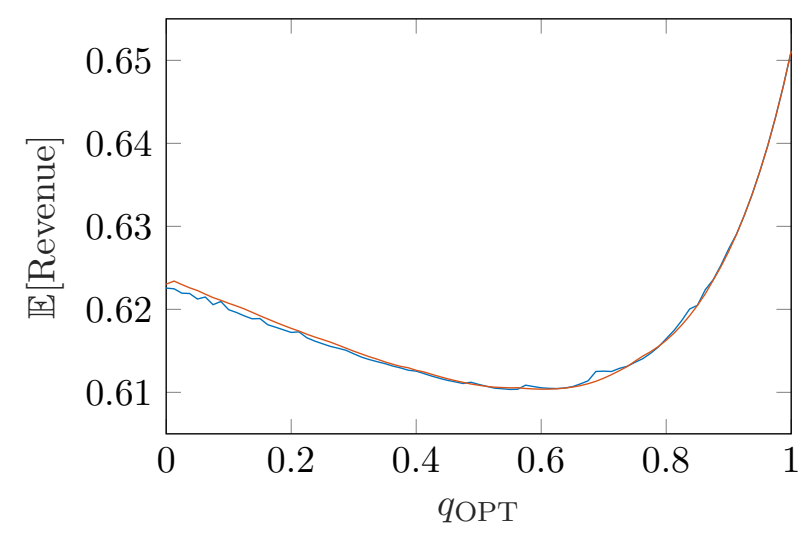

Figure 5: Comparison of derived upper bounds conditional on $q_{O P T}$. The expected revenue of the ERM mechanism for distributions obtained from primal solutions of (18) are shown in blue, while the expected revenue of the ERM mechanism for minimal distributions obtained from a grid-search approximation of $\eta\left(q_{O P T}\right)$ are shown in orange.

The results of our grid search are shown in Figure 5, juxtaposed with the upper bounds we obtain via solutions of (18). It appears that both curves approximate some convex shape on $[0,1]$. Since we do not have any approximation guarantees from our grid search, we are unable to tell if $\eta(\cdot)$ can or cannot provide stronger lower bounds than those derived via our primal solutions to (18) for any $q_{O P T}$ in general, but values we obtain via grid search do not improve on our upper bound of .61035.

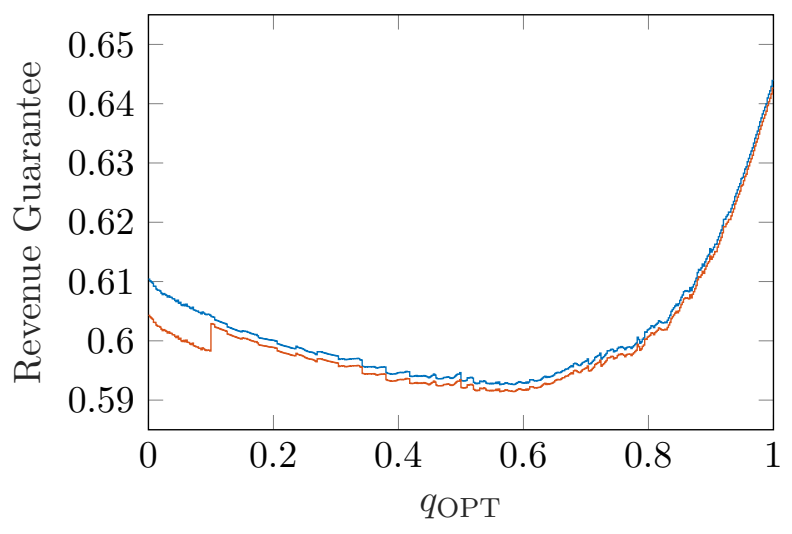

Figure 6: Results of computation of (23) for $n=50$ and $N=500$. The blue line shows the value of the objective for primal solutions found, while the orange line shows conditional lower bounds on the expected revenue of the auction. These results are corrected for tolerance of the MILP solver.

Finally, we compute (23) for $n=50$ and $N=500$, running our solver at $99.8 \%$ relative tolerance for $k>50$ and $99 \%$ relative tolerance for $k \leq 50$. The results of the computation are shown in Figure 6. Our results show that the ERM mechanism guarantees an expected revenue $\geq .5914$ times the optimal revenue. 


\section{Conclusion}

In this paper, we presented an MILP formulation to inspect the expected revenue of the ERM mechanism in the single item, single buyer, two sample setting. Working within this formulation has allowed us to greatly improve upon the known upper and lower bounds of the expected revenue guarantees of the ERM mechanism with two samples, and provided us with insights on what minimum revenue distributions may look like.

Despite the sheer number of binary variables involved, computations to certify our bounds were relatively cheap - on a ASUS ROG Zephyrus M (GU502GV) laptop, (18) for $n=80$ took approximately a day to compute, while the computations to solve (23) for $n=50$ and $N=500$ took around twelve days. Still, the exponential nature of the problem had become noticable around the values of $(n, N)$ we used. Therefore, we do not expect (18) and (23) to be feasibly solvable for significantly finer gauges, disallowing major improvements on the bounds we have provided by simply solving (18) and (23) for larger $n, N$.

That being said, it may be still possible to extract even stronger lower bounds within our framework. Lower bounds we may derive from solutions of (18) currently depend on the proof of Theorem 3.2. For fixed $n$, our estimation of how much the value of (18) overestimates $\alpha$ is $2 /(n-1)+(5 n-6) / n^{2}$. For $n=80$, this error estimate is $\lesssim .0869$, which means that our computations for (18) can only certify a lower bound of .5210. However, Figure 3 and Figure 5 hint that the actual error might in fact be much smaller than our estimate. Improving this estimate could then help certify stronger lower bounds on the revenue guarantees of the ERM mechanism with two samples.

There is also the question of what the minimal revenue curves for the ERM mechanism with two samples, conditional on $q_{O P T}$, actually look like. Figure 4 and Figure 5 suggest that 3-piecewise linear functions may be close to minimality, but we are currently unable to discern if they actually provide minimal instances. The separation of the two lines on $[0, .2]$ in Figure 5 hint that the answer is no, but the lines might have diverged simply because our search grid for 3-piecewise linear functions was not sufficiently fine. Even if this is the case, however, it might be that the minimum revenue curve is 3 -piecewise linear.

Finally, we note that our formulation should extend naturally to the setting with $\geq 3$ samples. However, in such an extension, the number of binary variables would blow up exponentially as the

number of samples increases for fixed number of intervals, $n$. This implies that the extension of (18) and (23) to a setting with $\geq 3$ samples might not be feasibly solvable. Still, for settings in which the performance of solvers do not depreciate too much, our techniques should be readily applicable.

\section{References}

[1] Alon, N., Babaioff, M., Gonczarowski, Y. A., Mansour, Y., Moran, S., and YehuDAYOFF, A. Submultiplicative Glivenko-Cantelli and uniform convergence of revenues. In Proceedings of the 31st International Conference on Neural Information Processing Systems (2017), NIPS'17, pp. 1655-1664.

[2] Babaioff, M., Gonczarowski, Y. A., Mansour, Y., and Moran, S. Are two (samples) really better than one? In Proceedings of the 2018 ACM Conference on Economics and Computation (2018), EC'18, p. 175. 
[3] Cole, R., And Roughgarden, T. The sample complexity of revenue maximization. In Proceedings of the 46th Annual ACM Symposium on Theory of Computing (2014), STOC'14, pp. 243-252.

[4] Daskalakis, C., and Zampetakis, M. More revenue from two samples via factor revealing SDPs. In Proceedings of the 21st ACM Conference on Economics and Computation (2020), EC'20, pp. 257-272.

[5] Devanur, N. R., Huang, Z., And Psomas, C.-A. The sample complexity of auctions with side information. In Proceedings of the 48th Annual ACM Symposium on Theory of Computing (2016), STOC'16, pp. 426-439.

[6] Dhangwatnotai, P., Roughgarden, T., and Yan, Q. Revenue maximization with a single sample. In Proceedings of the 11th ACM Conference on Electronic Commerce (2010), EC'10, pp. 129-138.

[7] Fu, H., Immorlica, N., Lucier, B., And Strack, P. Randomization beats second price as a prior-independent auction. In Proceedings of the 16th ACM Conference on Economics and Computation (2015), EC'15, p. 323.

[8] Gonczarowski, Y. A., and Nisan, N. Efficient empirical revenue maximization in singleparameter auction environments. In Proceedings of the 49th Annual ACM SIGACT Symposium on Theory of Computing (2017), STOC'17, pp. 856-868.

[9] Gonczarowski, Y. A., And Weinberg, S. M. The sample complexity of up-to- $\epsilon$ multidimensional revenue maximization. In IEEE 59th Annual Symposium on Foundations of Computer Science (2018), FOCS'18, pp. 416-426.

[10] Guo, C., Huang, Z., and Zhang, X. Settling the sample complexity of single-parameter revenue maximization. In Proceedings of the 51st Annual ACM SIGACT Symposium on Theory of Computing (2019), STOC'19, pp. 662-673.

[11] Huang, Z., Mansour, Y., And Roughgarden, T. Making the most of your samples. In Proceedings of the 16th ACM Conference on Economics and Computation (2015), EC'15, pp. $45-60$.

[12] Myerson, R. B. Optimal auction design. Mathematics of Operations Research 6, 1 (1981), $1-158$.

[13] Roughgarden, T., and Schrijvers, O. Ironing in the dark. In Proceedings of the 2016 ACM Conference on Economics and Computation (2016), EC'16, pp. 1-18. 